\title{
Office-Based Anesthesia in Oral and Maxillofacial Surgery-The American Model and Training
}

\author{
Payal Verma and Deepak G. Krishnan
}

\subsection{Introduction}

Anxiety and pain control has been the center of dental health provision from its inception. Dentists continue to practice some form of pain and anxiety control throughout the world. In the United States (US), Oral and maxillofacial surgery (OMS) has maintained a unique privilege where the surgeon is able to provide both anesthetic and surgical treatment for most of the procedures that are completed in a setting outside of the operation theater. The extensive, focused training received during OMS residency in the delivery of anesthesia, as well as continued education in anesthesia is central to this privilege. The art and science of alleviating pain and anxiety by providing office-based surgical treatment using various anesthetic techniques is associated with tremendous responsibility. Therefore, appropriate training along with local licensure, credentialing, and continuous education is of paramount importance.

While the Commission on Dental Accreditation (CODA) of the American Dental Association (ADA) stipulates standards in training OMS residents in anesthesia, American Association of Oral and Maxillofacial Surgeons (AAOMS) and state dental societies have developed parameters and protocols that are enforced among practicing OMSs, ensuring patient safety.

For the remainder of this chapter, the term ambulatory anesthesia would refer to the provision of anesthesia outside of a hospital operatory, such as a dental or an OMS office. Generally, this would entail provision of "open-airway" deep

P. Verma $(\bowtie)$

Oral \& Maxillofacial Surgery, University of Cincinnati Medical Center, OH, Cincinnati, USA

e-mail: vermapl@ucmail.uc.edu

D. G. Krishnan

Oral \& Maxillofacial Surgery, University of Cincinnati Medical Center, OH, Cincinnati, USA

Oral \& Maxillofacial Surgery, Cincinnati Children's Hospital and Medical Center, OH, Cincinnati, USA sedation with drugs delivered through an intra-venous (IV), intra-muscular (IM) or inhalational routes.

The American Society of Anesthesiology (ASA) recognizes four stages of anesthesia (Table 6.1) [1]. The most common forms of anesthesia used in an ambulatory office setting are mild-to-moderate sedation as well as deep sedation/general anesthesia with the use of inhalational and intravenous agents. As defined by ASA, moderate sedation/ analgesia (replacing the term "Conscious Sedation," which has been condoned) is a medication-induced depression of consciousness during which patients respond purposefully to verbal commands, either alone or often accompanied by light tactile stimulation. Other interventions are not required to maintain a patent airway, while spontaneous ventilation is adequate, and cardiovascular function is usually maintained. Whereas deep sedation/analgesia is a drug-induced depression of consciousness during which patients cannot be easily aroused but respond purposefully following repeated or painful stimulation. The ability to independently maintain ventilatory function may be impaired and may require assistance in maintaining a patent airway. However, it is important that the cardiovascular function is maintained.

Given that the process of sedation is a continuum, the patient's response is unpredictable. Hence, practitioners should be able to rescue the patients whose level of sedation becomes deeper than initially intended. An oral and maxillofacial surgeon is well trained in airway management and advanced life support.

\subsection{Brief History of Anesthesia in OMS}

Over several decades, Oral and maxillofacial surgeons have been at the forefront of anesthesia and pain control among all dental specialties and have been providing safe outpatient anesthesia.

Nitrous oxide was produced by Joseph Priestly and was termed as "laughing gas" by Humphrey Davy in eighteenth 
Table 6.1 Continuum of depth of sedation: definition of general anesthesia and levels of sedation/analgesia

\begin{tabular}{|c|c|c|c|c|}
\hline & $\begin{array}{l}\text { Minimal Sedation } \\
\text { Anxiolysis }\end{array}$ & $\begin{array}{l}\text { Moderate Sedation/ Analgesia } \\
\text { ("Conscious Sedation") }\end{array}$ & Deep Sedation/ Analgesia & General Anesthesia \\
\hline Responsiveness & $\begin{array}{l}\text { Normal response to } \\
\text { verbal stimulation }\end{array}$ & $\begin{array}{l}\text { Purposeful response to verbal } \\
\text { or tactile stimulation }\end{array}$ & $\begin{array}{l}\text { Purposeful response following } \\
\text { repeated or painful stimulation }\end{array}$ & $\begin{array}{l}\text { Unarousable even with } \\
\text { painful stimulus }\end{array}$ \\
\hline Airway & Unaffected & No intervention required & Intervention may be required & $\begin{array}{l}\text { Intervention often } \\
\text { required }\end{array}$ \\
\hline $\begin{array}{l}\text { Spontaneous } \\
\text { ventilation }\end{array}$ & Unaffected & Adequate & May be inadequate & Frequently inadequate \\
\hline $\begin{array}{l}\text { Cardiovascular } \\
\text { function }\end{array}$ & Unaffected & Usually maintained & Usually maintained & May be impaired \\
\hline
\end{tabular}

century [2, 3]. It was first used as an anesthetic agent in dental practice by Dr. Horace Wells [4] of Connecticut. He demonstrated the use of nitrous oxide for dental extractions that was aimed at the prevention of pain during surgery. Unfortunately, he was dismissed due to lack of medical background. His friend, William T G Morton [4], later demonstrated the use of ether vapors as anesthetic for the removal of a jaw tumor. Morton was therefore credited for the first anesthetic (October 16, 1846; also known as Ether Day). This solidified the foundation for the use of anesthetics in dental practice.

A prominent step forward in the provision of safe ambulatory anesthesia was the development and further refinement of the intravenous route of administration. It provided a safe route for rapid delivery and titration of medications along with giving the surgeon the ability to provide emergency medications. The practice of intravenous techniques allowed fast onset and recovery.

Adrian O. Hubbell [5] was critical in popularizing outpatient general anesthesia among OMS. He completed his training at Mayo Clinic and later demonstrated the safety of administering barbiturate anesthesia in an outpatient setting. He also advocated the utility and safety of recovering patients on their side or abdomen postoperatively to prevent aspiration of vomitus. Sylvan Shane [6] later reported on the use of intravenous drugs (benzodiazepines) for inducing sedation known as a "balanced" anesthetic. It was rapidly accepted due to its property of anxiolysis as a sole agent.

Another drug that became popular was methohexital [7] but was quickly overtaken by the introduction of Propofol [8], which is currently the most favored primary agent. In 1989, Propofol was officially approved for use, and its benefits related to the faster onset, improved recovery, and inherent antiemetic activity were soon recognized.

It became evident soon that to continue the privilege of administering outpatient anesthesia, it was important to receive adequate formal general anesthesia training and to maintain the highest standard of care. Today, Intravenous drug anesthesia is the mainstay of providing sedation in the outpatient setting of oral and maxillofacial surgery and has a history of safety [9].

\subsection{The Anesthesia Team}

A majority of the OMSs in the US work in private practice settings where ambulatory anesthesia is the mainstay of practice. This has led to the development of a unique office anesthesia model known as the Anesthesia Team model for OMS practice [10]. This was originally described as the operator-anesthetist model. That was a misnomer because in reality the entire team involved in patient care is trained to monitor and manage the patient under anesthesia in various capacities.

In a typical setting, it is the surgeon who performs the surgery and administers anesthesia. Apart from the surgeon, two or more other team members are present in the room (Fig. 6.1). At least one of these team members is required to be BLS (Basic life support) trained. Most commonly, one trained staff member observes the monitors, records drugs and vitals, and helps with i.v. drug administration, while another member acts as a surgical assistant. Assistant's job is to retract, suction, and irrigate during the surgery along with helping in supporting the airway (Fig. 6.2). Often surgeons employ a registered nurse with advanced cardiac life support (ACLS) training in their offices as well. The entire team is well versed with emergency equipment and is well trained to handle an airway emergency by providing positive pressure oxygen.

\subsection{AAOMS Office Anesthesia Guidelines}

AAOMS strives to ensure that its members throughout the country are in compliance with strict and mandated guidelines. It provides these guidelines through often-updated Office Anesthesia Evaluation document made available through the association. These guidelines are created by the Committee on Anesthesia (CAN) of the AAOMS. This committee uses the latest evidence-based recommendations and guidelines to make this document. This document is then vetted by the ASA to ensure that it gets the highest level of scrutiny and approval by that organization. This multilevel peer-review process of the guidelines is important for ensur- 


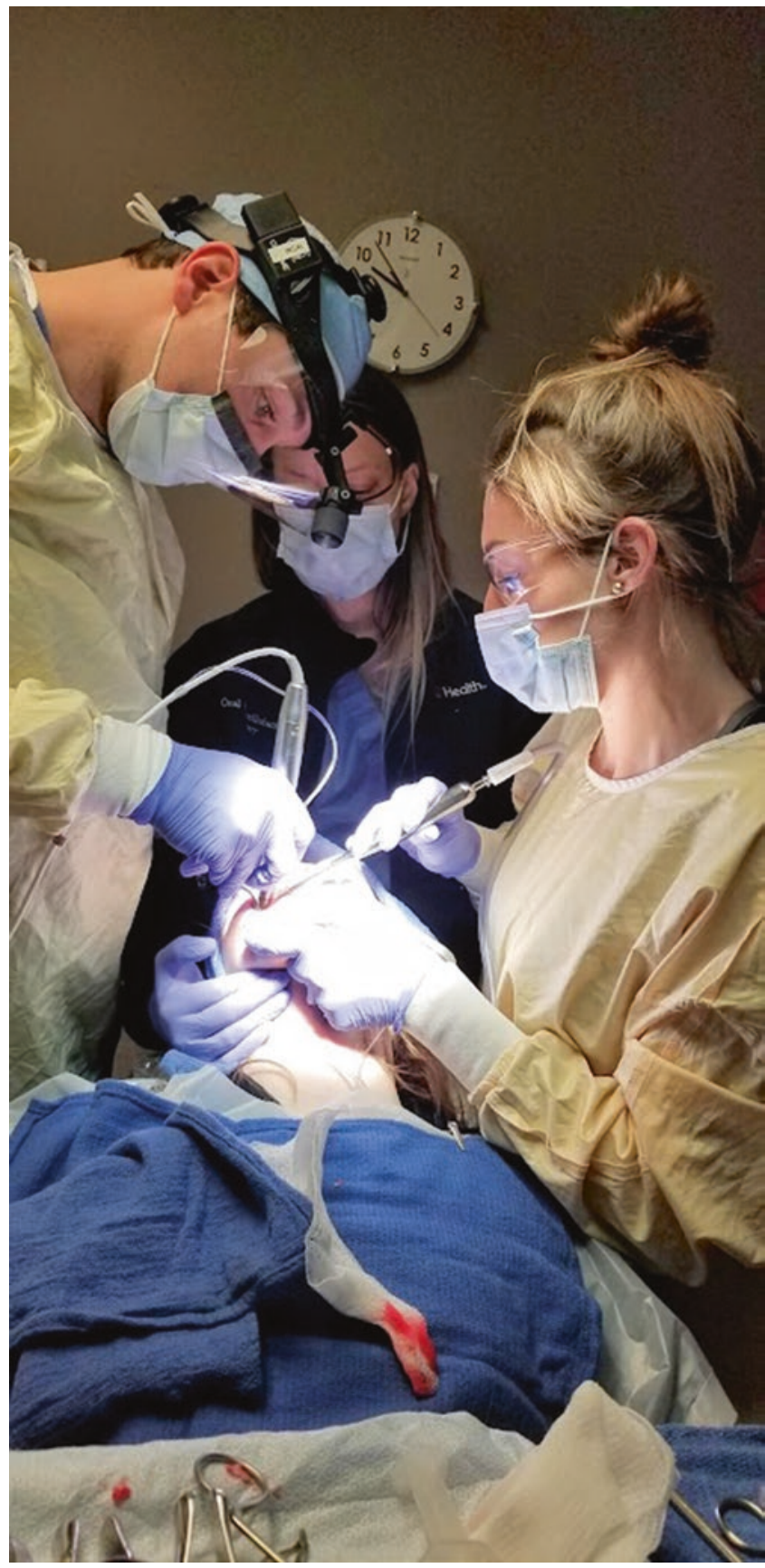

CAssociation of Oral and Maxillofacial Surgeons of India

Fig. 6.1 Example of the office anesthesia team. The surgeon provides the anesthesia and performs the procedure, one trained staff member supports the airway, and a second staff member is the surgical assistant

ing the safe delivery of anesthesia in the ambulatory OMS setting.

Every AAOMS member (close to 10,000 members) goes through a periodic office anesthesia evaluation process by peers to ensure that each office and team is current and well equipped to perform safe anesthetic practice in

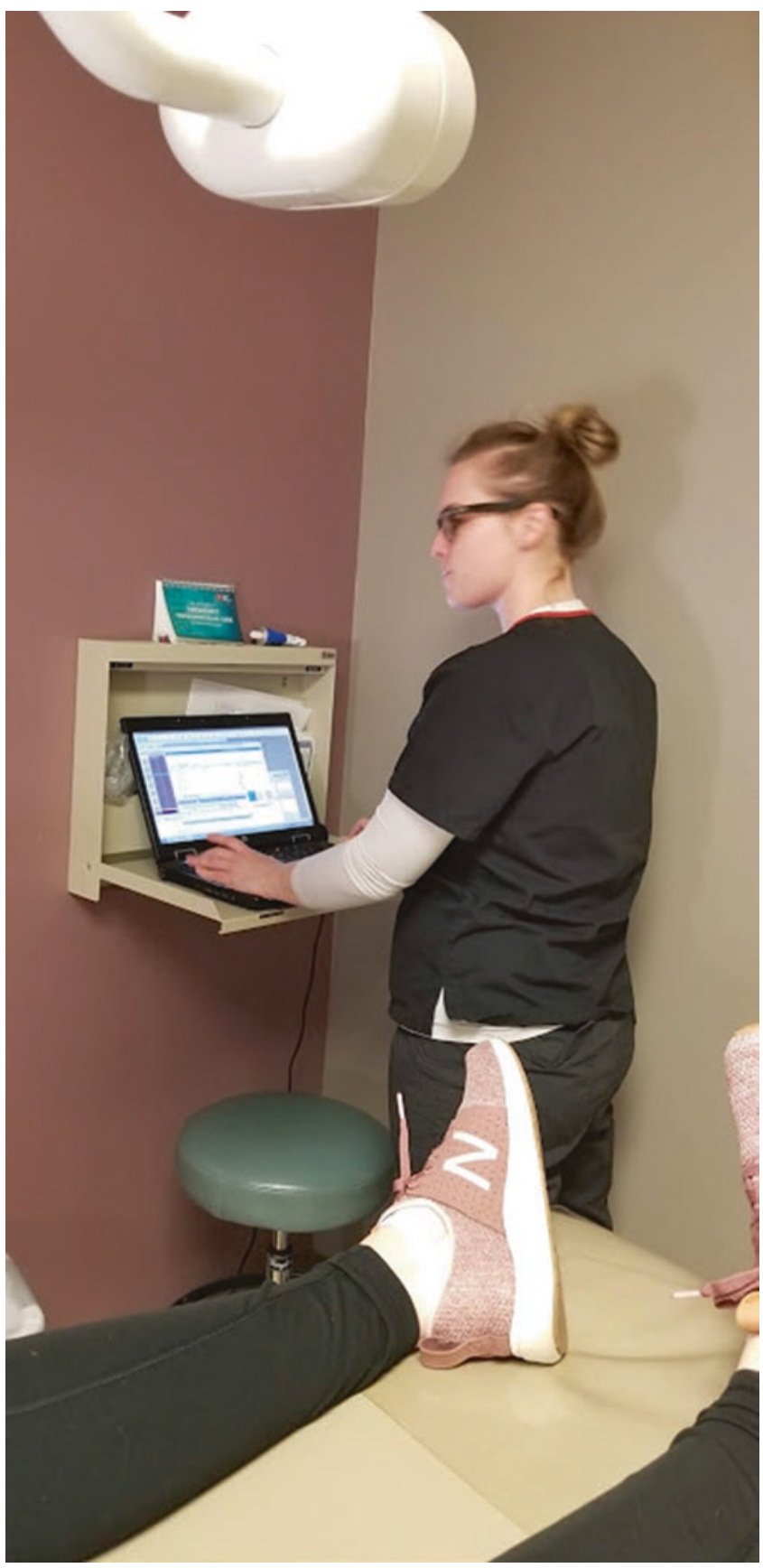

CAssociation of Oral and Maxillofacial Surgeons of India

Fig. 6.2 A dedicated registered nurse with ACLS training is often in charge of monitoring the patient under sedation

their setting (Fig. 6.3). Failure to pass such an evaluation leads to punitive action and inability to perform anesthesia in the office setting until corrective actions are taken. AAOMS, state dental societies, and third-party insurance carriers take this process seriously. Public safety cannot be compromised. 


\section{Sample Anesthesia On-site Inspection and Evaluation Form}

Date Sent to Society

\begin{tabular}{ll}
\hline Name of Practitioner Evaluated & General Anesthesia Permit Number (if applicable) \\
\hline Location(s) Inspected & Telephone Number \\
\hline Date(s) of Evaluation & Time of Evaluation
\end{tabular}

Names of Evaluators

\section{A. Personnel}

1. ACLS Certificate (Please have doctor's ACLS Certification available)

2. PALS Certificate (if appropriate)

3. Evidence of: one year advanced training in anesthesiology, Fellow of the American Dental Society of Anesthesiology, Diplomate of the American Board of Oral and Maxillofacial Surgery, eligible for examination by American Board of Oral and Maxillofacial Surgery, or Fellow of the American Association of Oral and Maxillofacial Surgeons

4. List of assisting staff's credentials/CV/training:

a.

b. c.

\section{B. Records}

Have available three charts of patients who have been treated in your office with intravenous sedation or general anesthesia.

1. An adequate medical history of the patient.

2. An adequate physical evaluation of the patient.

3. Anesthesia records showing: continuous monitoring of heart rate, blood pressure, and respiration using electrocardiographic monitoring and pulse oximetry, and ventilation by capnography. In patients undergoing inhalational anesthesia, preoperative and postoperative temperature monitoring, and if the procedure is longer than 30 minutes, continuous temperature monitoring.

4. Recording of monitoring every five minutes.

5. Evidence of continuous recovery monitoring, with notation of patient's condition upon discharge and person to whom the patient was discharged.

6. Accurate recording of medications administered, including amounts and time administered.

7. Records illustrating length of procedure.

8. Records reflecting any complications of anesthesia.

\section{Office Facility and Equipment}

1. Noninvasive Blood Pressure Monitor

\section{Electrocardiograph}

Fig. 6.3 Sample on site form for periodic office anesthesia evaluation. Available at www.aaoms.org 


\section{Defibrillator/Automated External Defibrillator}

\section{Pulse Oximeter}

5. Capnography equipment

6. Operating Theater

a. Is the operating theater large enough to accommodate the patient on a table or in an operating chair adequately?

b. Does the operating theater permit an operating team consisting of at least three individuals to move freely about the patient?

c. Does the operating theater allow easy access for emergency personnel and transportation equipment?

\section{Operating Chair or Table}

a. Does the operating chair or table permit the patient to be positioned so the operating team can maintain the airway?

b. Does the operating chair or table permit the team to alter the patient's position quickly in an emergency?

c. Does the operating chair or table provide a firm platform for the management of cardiopulmonary resuscitation?

\section{Lighting System}

a. Does the lighting system permit evaluation of the patient's skin and mucosal color?

b. Is there a battery-powered backup lighting system?

c. Is the backup lighting system of sufficient intensity and power for an adequate period of time to permit completion of any operation underway at the time of general power failure?

\section{Suction Equipment}

a. Does the suction equipment permit aspiration of the oral and pharyngeal cavities?

b. Is there a backup suction device available?

\section{Oxygen Delivery System}

a. Does the oxygen delivery system have adequate full-face masks and appropriate connectors, and is it capable of delivering oxygen to the patient under positive pressure?

b. Is there an adequate backup oxygen delivery system?

\section{Recovery Area (recovery area can be the operating theater)}
a. Does the recovery area have available oxygen?
b. Does the recovery area have available adequate suction?
c. Does the recovery area have adequate lighting?
d. Does the recovery area have adequate electrical outlets?
e. Can the patient be observed by a member of the staff at all times during the recovery period?

\section{Ancillary Equipment}

a. Is there a working laryngoscope complete with an adequate selection of blades, spare batteries and bulbs?

b. Are there endotracheal tubes and appropriate connectors?

c. Are there oral airways?

d. Are there any supraglottic airway devices?

e. Is there a tonsillar or pharyngeal type suction tip adaptable to all office outlets?

f. Are there endotracheal tube forceps?

g. Is there a sphygmomanometer and stethoscope?

h. Are there an electrocardioscope and defibrillator/automated external defibrillator?

Fig. 6.3 (continued) 
i. Is there a pulse oximeter?

j. Is there a capnography monitor?

k. Is there adequate equipment for the establishment of an intravenous infusion?

\section{Drugs}

1. Vasopressor drug available?

2. Corticosteroid drug available?

3. Bronchodilator drug available?

4. Muscle-relaxant drug available?

5. Intravenous medication for treatment of cardiopulmonary arrest available?

6. Narcotic antagonist drug available?

7. Benzodiazepine antagonist drug available?

8. Antihistamine drug available?

9. Antiarrhythmic drug available?

10. Anticholinergic drug available?

11. Coronary artery vasodilator drug available?

12. Antihypertensive drug available?

13. Mechanism of response for dantrolene (Dantrium)?

Overall Equipment - Facility

Adequate

Inadequate

Comments

Recommendations

Signature(s) of Evaluators

Printed Name(s) of Evaluators

10

Fig. 6.3 (continued) 


\subsection{Office Requirements}

The facilities where ambulatory anesthesia is provided in OMS practices are subject to stringent guidelines [11]. Specific building codes and local fire department regulations have to be followed for installation of remote gases. Proximity to a larger healthcare facility and access to emergency personnel are of paramount importance. Recommendations per Centers for Disease Control (CDC) and prevention on controls for exposure to anesthetic gases and other potential chemical hazards, as well as sterilization of the instruments and facilities are enforced. Office's fire safety and emergency protocols should be known by all staff and easily accessible for reference. Telephone numbers of the local ambulance service and nearest hospital should be clearly displayed and their location known to all office staff.

\subsection{Record Keeping}

Medical record keeping is paramount in current OMS practice. For the purpose of OMS procedures, proper electronic records have to be maintained. This includes preanesthesia assessment forms, i.e., history and physical evaluation, consent forms, intraoperative anesthesia records, operative notes as well as recovery records (Fig. 6.4). Anesthesia records typically consist of vital signs (recorded every $5 \mathrm{~min}$ ), type and amount of drugs administered with time of administration, start and end times of anesthesia and surgery, surgeon and other team members' names, and details of complications and management, if any.

\subsection{Office Anesthesia Evaluation Program and Parameters of Care}

Every practicing AAOMS member agrees to a uniform peer inspection of their properly equipped office and their techniques and preparation for managing emergencies and complications of anesthesia in the treatment of the OMS patient in their office or outpatient setting.

In 1975, the AAOMS established the Office Anesthesia Evaluation Program. This program was conceived, developed, and implemented by the AAOMS through its component state societies to benefit the public, whom its members serve. This ensures a level of safety to the practice of anesthesia in the OMS office, which is often outside a hospital or other healthcare facility. Since 1990, AAOMS Bylaws require official component societies' constitutions and bylaws to include provision for the fulfillment of an onsite office evaluation based on the AAOMS Office Anesthesia Evaluation Program as a prerequisite for active membership.
The office anesthesia evaluation is now required every 5-6 years for every AAOMS member.

AAOMS periodically offers an Office Anesthesia Evaluation Manual [12] with the following intent- "AAOMS Committee on Anesthesia developed the Office Anesthesia Evaluation Manual to provide information that could aid oral and maxillofacial surgeons in preparing themselves and their offices for the management of anesthetic complications. It presents scientific and clinical information and can serve as a reference for the practitioner." The most recent evaluation manual is available for purchase by the members in 2019.

In addition, a separate document called "AAOMS Parameters of Care" $[13,14]$ is intended to reflect practice considerations for 11 designated areas of oral and maxillofacial surgery and is an additional guide for AAOMS members to establish parameters of care for anesthesia in outpatient facilities. The most recent version of this document was published in 2017. The document provides complementary recommendations for the provision of safe sedation and anesthesia in the office. Members are encouraged to review both documents, which provide essential information on contemporary OMS office anesthesia practice and practice accordingly.

\subsection{AAOMS National Simulation Program}

AAOMS recognizes that the provision of anesthesia at an OMS office is a team effort. It expects each member of the team-from the surgeon to the assistants-to be highly trained and qualified to address every anesthesia-related situation that may occur during a surgical procedure. This training is initiated in OMS residencies but is continuous throughout the surgeon's career, mandated by continuing education and team exercised. Similar to professional sports teams, successful oral and maxillofacial surgery teams train as a unit to ensure they can seamlessly and quickly manage an unexpected airway emergency. The most recent AAOMS offering to help the office anesthesia team partners with advanced simulation technology making it possible to train surgeons and staff by simulating a full range of real-life emergency airway situations [15]. This program allows participants to practice and master critical techniques for administering and monitoring office-based anesthesia; a standardized approach that ensures that every participant experiences the same simulated events. The program allows for evaluation of each participant's performance and can pinpoint those areas that may benefit from additional training. The three-module program offers oral and maxillofacial surgeons and their staff an effective method of assessing their readiness to meet an office anesthesia emergency situation by practicing these in a low-risk, high-stake simulation scenario. 


\section{Sedation and Anesthesia Record}

\begin{tabular}{|c|c|c|c|c|c|c|c|}
\hline \multicolumn{2}{|l|}{ Patient } & Date & Age & NPO & ASA & Surgeon & Anesthetist \\
\hline ID\# & & & & & 123 & & \\
\hline $\begin{array}{l}\square \text { Premed } \\
\square \text { Equipment Check }\end{array}$ & Dx: & Weight & Height & BMI & Airway & Surgeon Asst. & Anesthetist Asst. \\
\hline $\begin{array}{l}\square \text { Time Out } \\
\text { Preoperative start time }\end{array}$ & Tx: & & & & $\begin{array}{r}\text { Mallampati } \\
1234\end{array}$ & & \\
\hline
\end{tabular}

Preoperative start time:

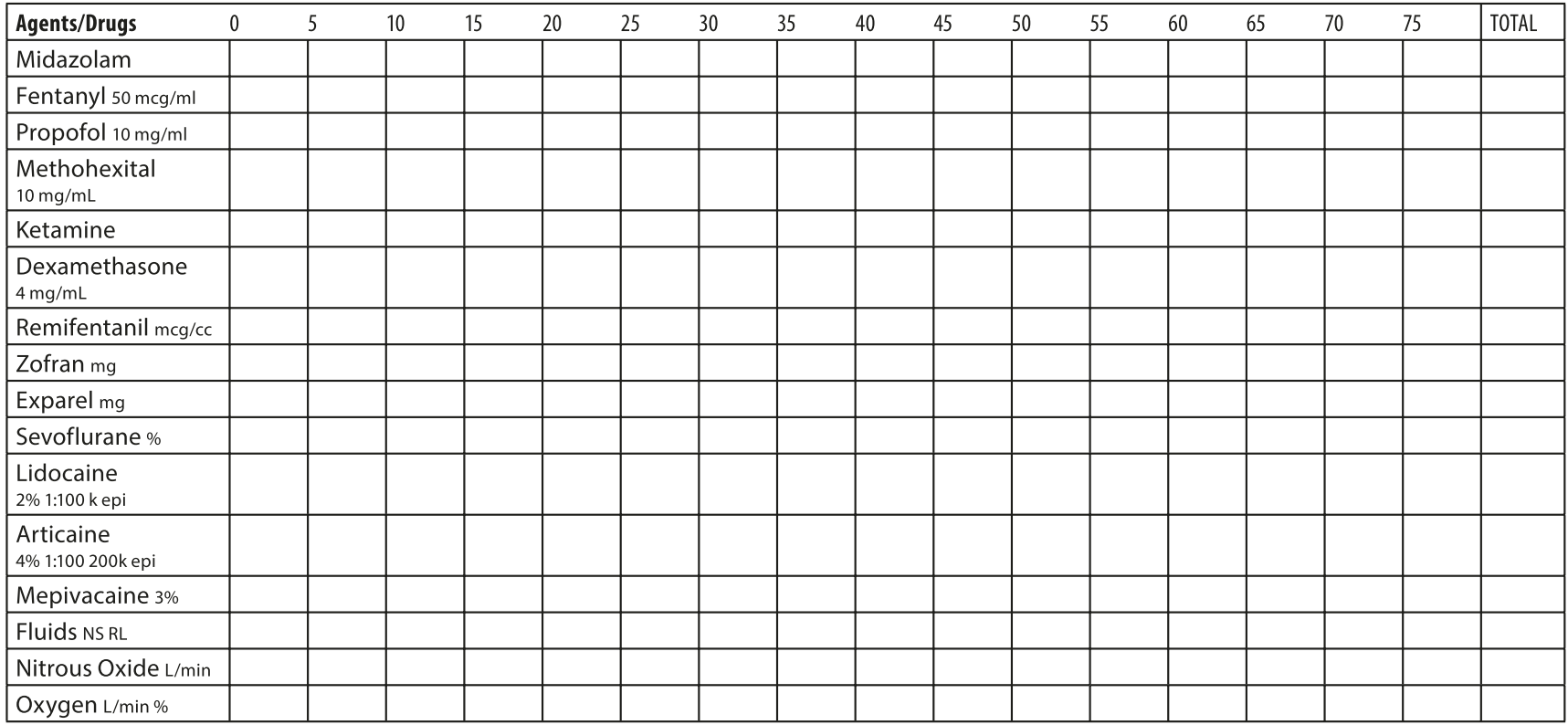

\begin{tabular}{|l|}
\hline \multicolumn{2}{|c|}{ MONITORS } \\
\hline$\square$ Auto BP R L \\
\hline$\square$ ECG (Lead II) \\
\hline$\square$ Pulse Oximeter \\
\hline$\square$ Stethoscope \\
\hline$\square$ Capnograph \\
\hline$\square$ BIS \\
\hline$\square$ Temp \\
\hline \multicolumn{1}{|c|}{ SYMBOLS } \\
\hline SBP $\vee$ \\
DBP $\wedge$ \\
Pulse $\bullet$ \\
Resp $\bigcirc$ \\
Anes x \\
Anes $\underline{x}$ \\
Surg $\odot$ \\
Surg $\bigodot$ \\
\hline \multicolumn{2}{|c|}{ POSITION } \\
\hline$\square$ Reclined \\
\hline$\square$ Supine \\
\hline
\end{tabular}

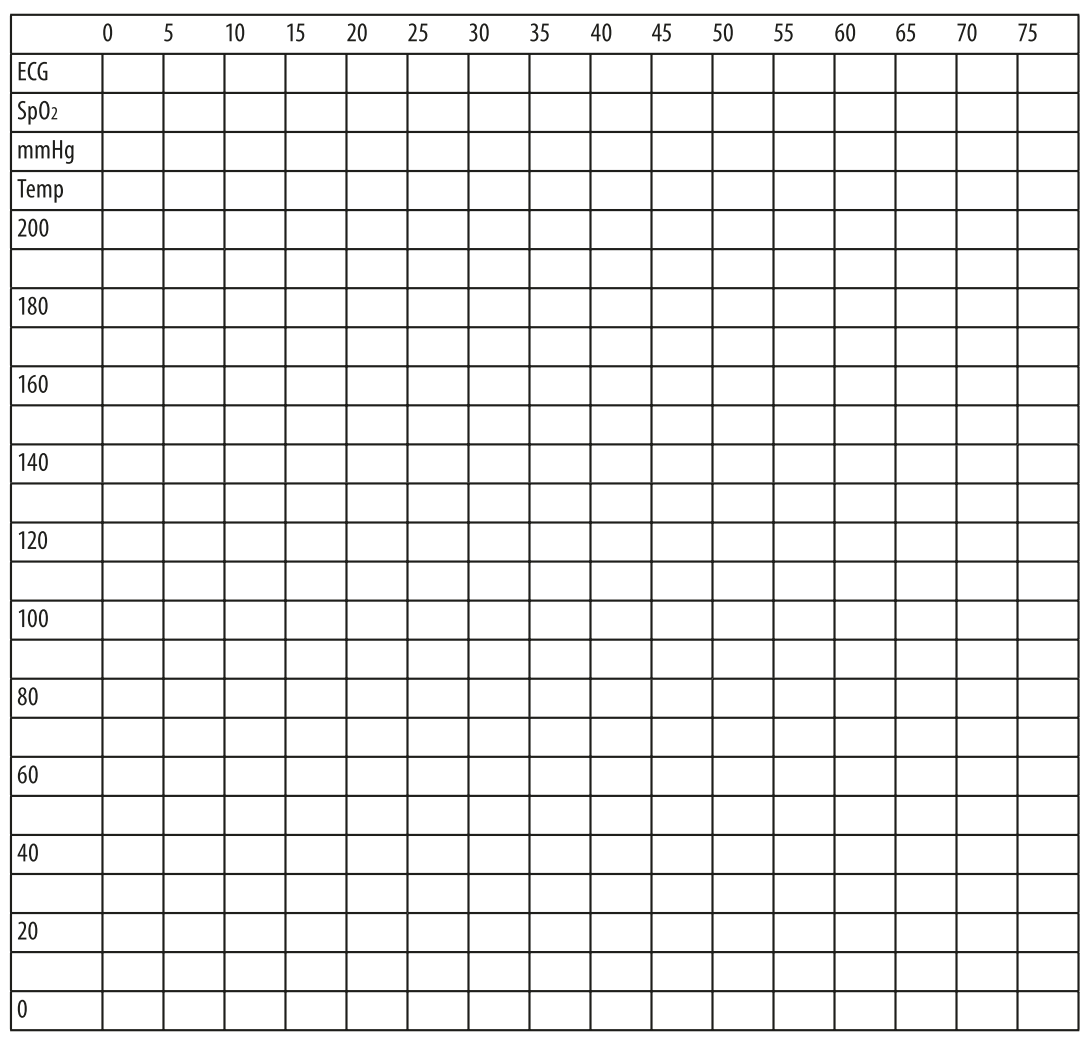

Dr. Signature

\begin{tabular}{|l|l|}
\hline \multicolumn{2}{|c|}{ IV } \\
\hline$\square$ 22G Catheter & $x$ \\
\hline$\square$ 20G Catheter & $x$ \\
\hline$\square$ & $x$ \\
\hline$R$ Antecubital & $x$ \\
\hline$\square$ Radial & $x$ \\
\hline$L \quad$ Dorsum hand & $x$ \\
\hline$\square$ & $x$ \\
\hline \multicolumn{2}{|c|}{ AlRWAY } \\
\hline$\square$ Nasal Cannula \\
\hline$\square$ Nasal Mask \\
\hline$\square$ Mask \\
\hline$\square$ Nasopharyngeal \\
\hline$\square$ Oral \\
\hline$\square$ LMA \\
\hline$\square$ ET Tube \\
\hline
\end{tabular}

Comments

Fig. 6.4 Sample sedation and anesthesia record form. Available at www.aaoms.org 


\subsection{Dental Anesthesia Assistant National Certification Examination (DAANCE)}

The practice of office-based anesthesia depends largely on the skillset of the assistants in the office as much as it does on the surgeon leading the team. In an effort to elevate the education and knowledge base of the other team members, a program called the Dental Anesthesia Assistant National Certification Examination (DAANCE) was implemented nationwide. This is a continuing education program that comprises $36 \mathrm{~h}$ of self-education and a computerized exam to be completed in 6 months. This course is designed primarily for OMS assistants. Upon successful completion of the final examination, certification is provided.

The course is designed to cover important topics, including general review of basic sciences, patient's medical history evaluation, basic knowledge on anesthetic drugs and techniques, role of anesthesia equipment and monitoring, and covers basic maneuvers to handle office anesthesia emergencies.

This course is available to allied staff all year round to register and complete. More and more states in the US are now mandating courses like DAANCE or others to all team members in an effort to keep the practice of ambulatory anesthesia team model safe and viable in the OMS office.

\subsection{Anesthesia Training during OMS Residency}

OMS trainees in the US have a rigorous curriculum in anesthesia. The Commission on Dental Accreditation (CODA) of the American Dental Association (ADA) is the independent body that accredits all residency training programs. This accreditation is based on a peer-review process that enforces adherence to training standards created and vetted by the CODA. The educational standards for anesthesia read as follows:

4-3.1... Anesthesia Service: The assignment must be for a minimum of 5 months, should be consecutive, and one of these months should be dedicated to pediatric anesthesia. The resident must function as an anesthesia resident with commensurate level of responsibility.

Intent: The pediatric portion could include Pediatric intensive care unit (PICU), Neonatal intensive care unit (NICU), pediatric anesthesia service, or ambulatory pediatric anesthesia. Oral and maxillofacial surgery residents rotating on the anesthesia service have levels of responsibility identical to those of the anesthesia residents, and abide by the anesthesia department's assignments and schedules. Part of this time can be during medical school as long as oral and maxillofacial surgery trainee functions at the anesthesia resident level.

4-9... The off-service rotation in anesthesia must be supplemented by longitudinal and progressive experience throughout the training program in all aspects of pain and anxiety control. The ambulatory oral and maxillofacial anesthetic experience must include the administration of general anesthesia/deep sedation for oral and maxillofacial surgery procedures to pediatric, adult, and geriatric populations, including the demonstration of competency in airway management. Examples of evidence to demonstrate compliance may include:

- Resident's anesthetic log.

- Clinical tracking system.

- Anesthesia records.

- Oral and Maxillofacial Surgery Benchmarks.

4-9.1... The cumulative anesthetic experience of each graduating resident must include administration of general anesthesia/deep sedation for a minimum of 300 cases. This experience must involve care for 50 patients younger than 13. A minimum of 150 of the 300 cases must be ambulatory anesthetics for oral and maxillofacial surgery outside of the operating room. Intent: The cumulative experience includes time on the anesthesia rotation as well as anesthetics administered while on the oral and maxillofacial surgery service. Locations for ambulatory anesthesia may include dental school clinics, hospital clinics, emergency rooms, and oral and maxillofacial surgery offices.

Examples of evidence to demonstrate compliance may include:

- Resident's anesthetic log.

- Clinical tracking system.

- Anesthesia records.

- Oral and Maxillofacial Surgery Benchmarks.

4-9.2 ...In addition to general anesthesia/deep sedation, the residents must obtain extensive training and experience in all sedation techniques.

Examples of evidence to demonstrate compliance may include:

- Detailed curriculum plans

- Patient charts

- Simulation experience

4-9.3 ...The clinical program must be supported in part by a core comprehensive didactic program on general anesthesia, deep sedation, and other methods of pain and anxiety control. The didactic program must include lectures and seminars emphasizing:

- Patient evaluation

- Risk assessment

- Anesthesia and sedation techniques

- Monitoring and

- The diagnosis and management of complications

4-9.4 ...Advanced Cardiac Life Support (ACLS) must be obtained in the first year of residency and must be maintained throughout residency training and thereafter. 
Examples of evidence to demonstrate compliance may include:

- ACLS certification records and cards

4-9.5... Each resident must be certified in Pediatric Advanced Life Support (PALS) prior to completion of training.

Examples of evidence to demonstrate compliance may include:

- PALS certification records and cards

Adherence to these standards is strictly enforced ensuring a standardized education in anesthesia to each OMS resident in training in the US.

\subsection{Preanesthetic Evaluation of the Patient}

The OMS planning an office-based anesthetic is trained to do a thorough preoperative evaluation of the patient to deem them fit for office anesthesia. Just as an anesthesiologist would evaluate the patient for systemic disease, functional capacity and medications along with a detailed anesthetic history and physical examination to classify every patient into an American Society of Anesthesiologists (ASA) status (Table 6.2) [16], every OMS would do the same for formulating an anesthetic plan for the patient.

Ideally, ASA I and II patients are best suited for officebased anesthetics. Careful preanesthetic patient evaluation is critical in choosing the type and venue for the anesthetic. This evaluation has the following essential steps:

- Reviewing and understanding patient's medical, family, social, surgical, and anesthetic history through questionnaire and interview.

- Full physical examination of all systems.

This detailed preop evaluation gives the OMS the knowledge to classify all according to the ASA classification and adequately plan for an appropriate anesthesia technique.

\subsection{Monitoring}

Provision of anesthesia in the OMS office requires that monitoring is done according to the ASA standards and more.

This includes the following minimal time-stamped monitoring and documentation [17].

1. Noninvasive monitoring of blood pressure every $5 \mathrm{~min}$

2. Continuous ECG monitoring

3. Continuous pulse oximetry

4. Continuous end-tidal $\mathrm{CO} 2$ monitoring of ventilatory effort
Table 6.2 ASA physical status classification system.

\begin{tabular}{|c|c|c|}
\hline$A S A I$ & $\begin{array}{l}\text { A normal healthy } \\
\text { patient }\end{array}$ & $\begin{array}{l}\text { Healthy, nonsmoking, no or minimal } \\
\text { alcohol use }\end{array}$ \\
\hline ASA II & $\begin{array}{l}\text { A patient with } \\
\text { mild systemic } \\
\text { disease }\end{array}$ & $\begin{array}{l}\text { Mild diseases only without substantive } \\
\text { functional limitations. Examples include } \\
\text { (but not limited to): Current smoker, } \\
\text { social alcohol drinker, pregnancy, } \\
\text { obesity }(30<\text { BMI }<40 \text { ), well- } \\
\text { controlled DM/HTN, mild lung disease }\end{array}$ \\
\hline ASA III & $\begin{array}{l}\text { A patient with } \\
\text { severe systemic } \\
\text { disease }\end{array}$ & $\begin{array}{l}\text { Substantive functional limitations; one } \\
\text { or more moderate-to-severe diseases. } \\
\text { Examples include (but not limited to): } \\
\text { Poorly controlled DM or HTN, COPD, } \\
\text { morbid obesity (BMI } \geq 40 \text { ), active } \\
\text { hepatitis, alcohol dependence or abuse, } \\
\text { implanted pacemaker, moderate } \\
\text { reduction of ejection fraction, ESRD } \\
\text { undergoing regularly scheduled dialysis, } \\
\text { premature infant PCA < } 60 \text { weeks, } \\
\text { history ( }>3 \text { months) of MI, CVA, TIA, } \\
\text { or CAD/stents. }\end{array}$ \\
\hline$A S A I V$ & $\begin{array}{l}\text { A patient with } \\
\text { severe systemic } \\
\text { disease that is a } \\
\text { constant threat to } \\
\text { life }\end{array}$ & $\begin{array}{l}\text { Examples include (but not limited to): } \\
\text { Recent (<3 months) MI, CVA, TIA, or } \\
\text { CAD/stents, ongoing cardiac ischemia } \\
\text { or severe valve dysfunction, severe } \\
\text { reduction of ejection fraction, sepsis, } \\
\text { DIC, ARD or ESRD not undergoing } \\
\text { regularly scheduled dialysis }\end{array}$ \\
\hline$A S A V$ & $\begin{array}{l}\text { A moribund } \\
\text { patient who is not } \\
\text { expected to } \\
\text { survive without } \\
\text { the operation }\end{array}$ & $\begin{array}{l}\text { Examples include (but not limited to): } \\
\text { Ruptured abdominal/thoracic aneurysm, } \\
\text { massive trauma, intracranial bleed with } \\
\text { mass effect, ischemic bowel in the face } \\
\text { of significant cardiac pathology or } \\
\text { multiple organ/system dysfunction }\end{array}$ \\
\hline$A S A V I$ & $\begin{array}{l}\text { A declared } \\
\text { brain-dead patient } \\
\text { whose organs are } \\
\text { being removed for } \\
\text { donor purposes }\end{array}$ & \\
\hline
\end{tabular}

Developed By: ASA House of Delegates/Executive Committee

5. Ability to measure temperature, i.e., very essential in a setting where Malignant Hyperthermia $(\mathrm{MH})$ triggering agents are in use.

Majority of OMS offices now use monitor units (Fig. 6.5) that interface directly with an electronic health record. The entire team is clued into monitoring with a staff member often dedicated to only monitoring the patient.

All patients receive supplemental oxygen before, during, and after the procedure. Most facilities have a separate recovery area with the same monitors, although there are several offices that recover in the operatory. The goal is the improvement of efficacious monitoring to establish the safe performance of anesthetic without the need for a separate anesthesia provider. It is imperative to have a meticulously trained team to allow for continuous monitoring, thus facilitating immediate recognition of changes in patient's vital signs. A properly evaluated and selected patient is unlikely to have a catastrophe in the OMS anesthetic, but when an untoward event does occur, it is often a respiratory event. 


\subsubsection{Methods for Monitoring Ventilation}

Data available from closed claims cases from the OMS National Insurance Corporation (OMSNIC) allow us as a specialty to audit the near-misses and tragic outcomes from our offices. The data suggest a strong correlation between airway-related failure and adverse events in the open-airway anesthetic techniques. The emphasis is on monitoring of

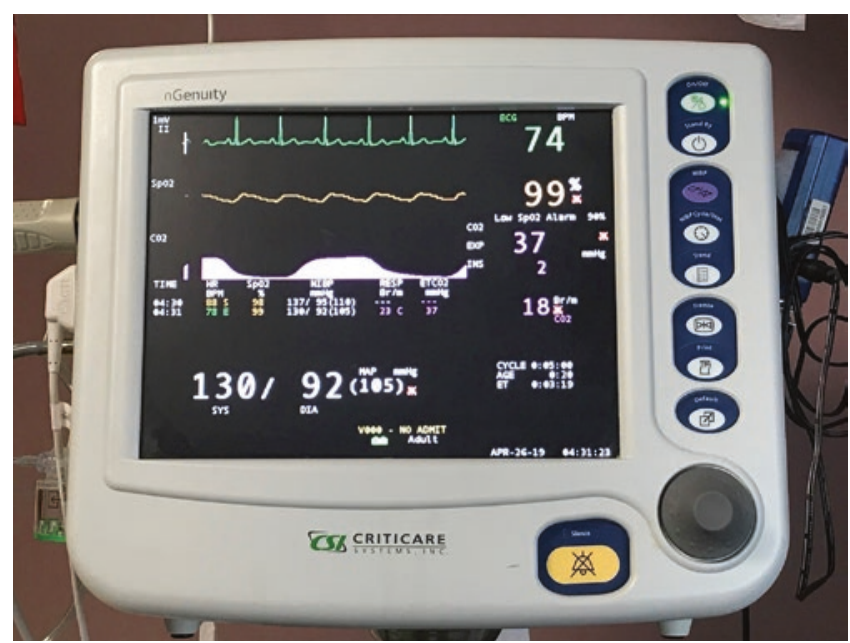

CAssociation of Oral and Maxillofacial Surgeons of India

Fig.6.5 Example of monitor commonly used in oral and maxillofacial surgery anesthetic administration. The single unit clearly displays the electrocardiogram, noninvasive blood pressure monitor, oxygen saturation, and end-tidal carbon dioxide both ventilation and oxygenation to allow adequate monitoring of the respiratory system during anesthesia. This can be achieved by using a pulse oximeter, which is a device that measures oxygen saturation and provides an indirect indication of oxygenation. Recently, AAOMS recommended a newer ASA standard monitoring of ventilation with continuous capnography. Although chest movements are a reliable method but are least exact, hence the addition of capnography. Most OMSs use a pretracheal/precordial stethoscope (Fig. 6.6) to auscultate while the patient is sedated.

Historically, pulse oximetry has been used for respiratory monitoring. But due to its limitation in lacking real-time responsiveness to major respiratory events like airway obstruction or respiratory depression, there was an imminent need to incorporate a different parameter to provide a real-time assessment. Thus was developed capnography [18], which is the noninvasive measurement of the partial pressure of carbon dioxide $(\mathrm{CO} 2)$ from the airway during inspiration and expiration. It can provide real-time sensitivity to early changes in ventilation and is reliable even in low-perfusion states.

Although, in the nonintubated sedated patient, there is loss of $\mathrm{CO} 2$ through the nose and mouth, and thus requires modification of the collection cannula, it still provides valuable clinical information on patient's ventilation during sedation.

It cannot be stressed enough how critical it is to manage intraoperative airway and monitor respiratory status. Ventilation is monitored by inspection of chest movement by the assistants, monitoring of capnography as well as auscul-
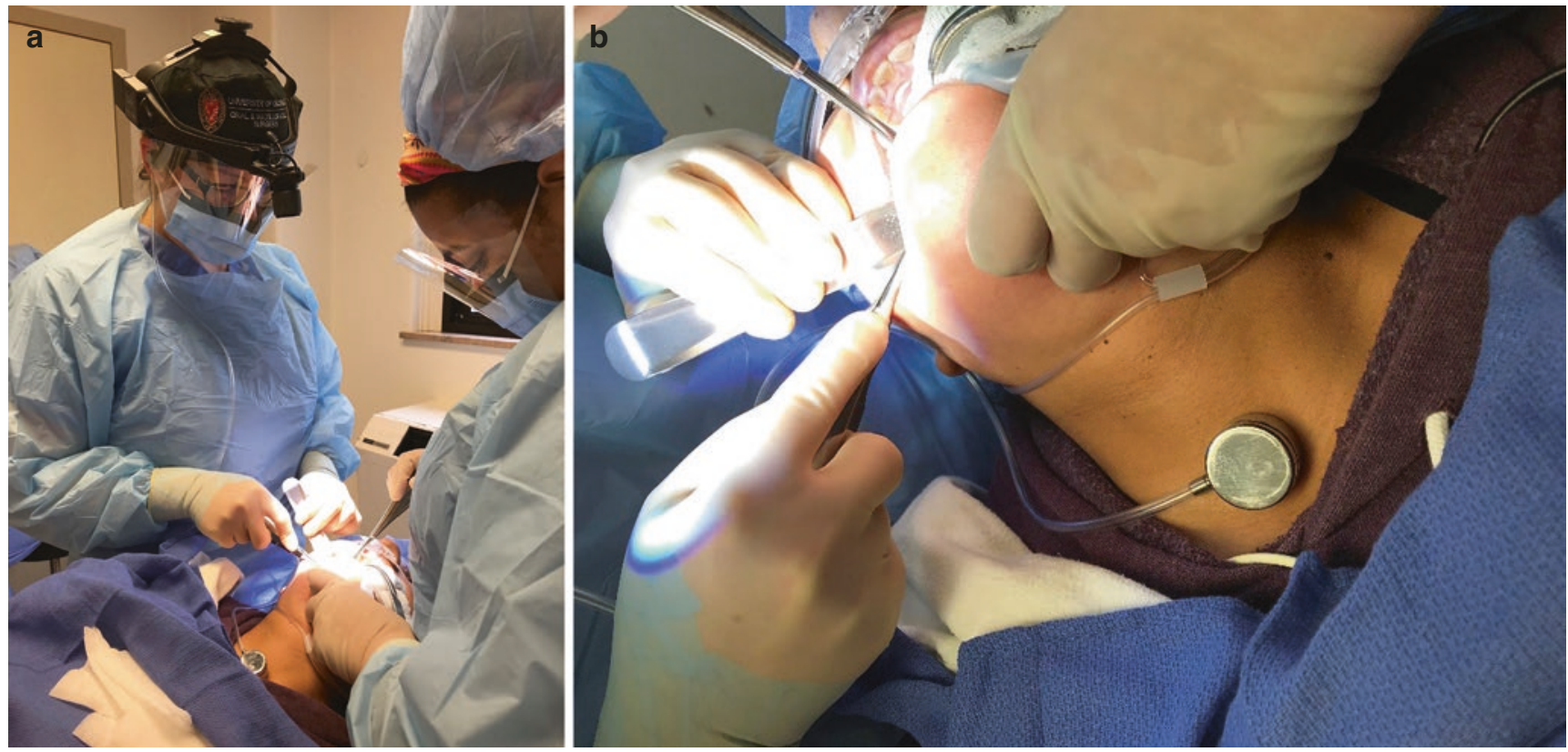

CAssociation of Oral and Maxillofacial Surgeons of India

Fig.6.6 (a, b) Pretracheal/precordial stethoscope used by the surgeon to auscultate while the patient is sedated 
tation with a precordial stethoscope. The newer Bluetooth models of the precordial stethoscope allow wireless speakers that allow the entire team to listen to the patient's respiratory patterns.

Now with multiple modalities such as a real-time capnography tracing, listening to breath sounds with high fidelity, OMS team has an opportunity to assess and act promptly on potential airway threats even before the adverse respiratory event comes to light. With these available modalities, along with surgeon's experience and developed senses, anticipation and prediction of an impending laryngospasm or bronchospasm prompts the team to act preemptively. Even before a drop in oxygen saturation or loss of waveform on capnography becomes apparent, both the depth and rate of breathing as well as the cessation of breathing can be promptly recognized.

\subsection{Airway Armamentarium}

Despite several preventive modalities available to the OMS performing anesthesia, emergencies are bound to happen. The unit must be prepared to handle airway and other emergencies. All the anesthesia providers should be exceptionally familiar with emergency airway equipment that must be readily accessible in both the operating suites and the recovery area [11].

Emergency airway equipment must include full face mask, bag-valve-mask device capable of providing positive pressure ventilation, oral and nasopharyngeal airways, supraglottic airway devices, endotracheal tubes, and laryngoscope and cricothyrotomy kit. All these equipment should be available in both pediatric and adult sizes. The entire staff should have periodic in-office training to familiarize themselves with the equipment and their use. Apart from emergency airway instruments, all offices are required to have either a basic monophasic defibrillator or automated external defibrillator.

\subsection{Oxygen and Supplemental Gas Delivery System}

In addition to oxygen, most OMS offices utilize other gases such as nitrous oxide and inhalational anesthetics as well as air. The oxygen delivery system should be able to deliver metered oxygen to the patient under positive pressure. A standard fail-safe mechanism along with scavenging system should be part of all gas delivery machines installed in the office. This prevents delivery of any hypoxic amount of gases. To prevent the accidental administration of an incorrect gas, the gas outlets used should be pin-indexed. The gas delivery machines also are mandated to have periodic cali- bration records, which should be maintained with proper documentation stored at a known location. Anesthesia equipment, including ventilators, are similarly required to have a periodic inspection by biomedical engineers. The OMS office is responsible for ensuring this happens and the office anesthesia evaluation is a mechanism to keep a check on this.

\subsection{Suction Equipment}

As trivial as it may seem, the office suction unit (central and portable) is an integral part of both the normal and emergency functions of that office. A portable suction unit must be readily accessible in the operatory and recovery area in case of unanticipated power failure or central suction pump failure. Again, routine maintenance of the suction system and its documentation is required.

\subsection{Recovery Room}

Most OMS offices have a postoperative recovery unit separate from the operatory that allows unobstructed patient observation. The design of this area must allow resuscitation and management of the patient during an emergency. The recovery area should have a suction system, oxygen delivery system, all appropriate monitors and emergency equipment as detailed earlier. It is also the responsibility of the recovery nurse to document the patient's recovery from anesthetic to deem their fitness to leave the office. A recovery checklist such as the Modified Aldrete Scale is useful in making this determination.

\subsection{Techniques in Anesthesia}

Training in anesthesia exposes the oral and maxillofacial surgeon to the different modalities of anesthesia from light to deep sedation. As described before, levels of anesthesia are a continuum (Table 6.1). The effects of different drugs and techniques may vary between individuals. Patients may achieve deeper or lighter levels of anesthesia than anticipated based on their unique metabolic responses to the different anesthetics administration. The OMS team is prepared to anticipate these differences in responses and intervene accordingly.

The techniques utilized to get to different levels of anesthesia include inhalational anesthesia, oral anesthetic technique, parenteral anesthesia, and local anesthesia. Regardless of the technique employed, the goals of each of these techniques are to reduce anxiety, establish cooperation, amnesia and analgesia, ensuring comfort and hemodynamic stability. In order to choose the level of anesthesia, one should con- 
sider the age of the patient, level of anxiety and ability to cooperate, detailed medical history, and prior anesthesia experience.

\subsubsection{Enteral Anesthesia}

Oral or nasal route of delivery of drugs is the least threatening route of administration of anesthesia. While some anxiolysis is achieved via this modality, it is rarely used to achieve deeper levels of anesthesia. Often the oral route of sedation is used as a premedication prior to IV access in an uncooperative patient. Various ingestives such as syrups, popsicles, needleless syringes, other creative methods are employed to get the patient to consume the medication. Dosing is adjusted in such a way that a higher dose of the drug may be administered in a limited volume to prevent the likelihood of aspiration. Despite that, a crying child or a coughing adult may end up aspirating the medication. The other disadvantages of this technique include a slow onset and variable response to the action of the drug as well as an unpredictable recovery pattern. In an uncooperative patient, patient compliance will limit oral or nasal administration of any drug. Additionally, oral dosing is often empiric with almost predictable erratic absorption. Titration of the drug is difficult via this route.

Yet, it remains a convenient mode of administration, requiring very little training to deliver, and any adverse reactions are slow to the onset and usually less severe and manageable. In the US, a medical provider providing oral sedation in the office does not have an increase in their malpractice premiums because of this practice. In contrast, practitioners of general anesthesia have higher malpractice coverage due to the inherent risks of that practice.

An ideal oral medication must be able to provide rapid absorption, must have a rapid onset, and must have a high therapeutic index allowing rapid and predictable recovery.

The following are the drugs most commonly used in oral sedation techniques:

- Benzodiazepines

- Histamine blockers

- Opioids

- Scopolamine

- Chloral hydrate

- Barbiturates

- Alpha agonists-Dexmedetomadine, Clonidine

Oral sedation techniques are primarily useful in the OMS as a premedication tool. An orally sedated patient is likely to accept a mask or an IV for further induction of anesthesia. Several minor procedures, however, can be performed with oral sedation and good local anesthesia alone.

\subsubsection{Parenteral Anesthesia: Intravenous Anesthesia and Inhalational Anesthesia}

Hubbell [5] was known to promote the use of intravenous thiopental anesthesia. It was eventually replaced by the shorter-acting barbiturate methohexital. This became the primary agent used for office-based anesthesia for multiple decades. A shortage in supply of methohexital and an increased use of Propofol [8] in the operating theater setting prompted an adaptation of the use of that drug in the officebased anesthetic technique. The basic technique for deep sedation and general anesthetic in the nonintubated patient in the OMS office typically employs a cocktail of medications under strict ASA-mandated guidelines for monitoring with the personnel as described earlier.

These medications include, but are not limited to, the following:

- A benzodiazepine such as Midazolam for anxiolysis, amnesia, sedation, and hypnosis

- A potent narcotic analgesic such as Fentanyl or Remifentanil

- A potent non-narcotic analgesic such as Ketamine

- A sedative hypnotic such as Propofol. It also acts as an adjunct antiemetic agent.

- Adjuvant drugs such as dexamethasone for prevention of postoperative edema and nausea and vomiting.

- Toradol or i.v. Tylenol for adjunct pain control

Most of these medications are characterized by their ability to have a rapid onset and offset. Medications such as Propofol rapidly redistribute and metabolize and are eliminated from the system. The combination of these medications often results in a cumulative effect of sedation and hypnosis in addition to central analgesia. Discomfort from the administration of local anesthesia is often obtunded by this level of sedation. Patients maintain their ventilation and careful monitoring of their vital signs and protection of their airway allows for smooth sedation. The surgeon who is performing the procedure is in close proximity to the patient's airway understanding its minute undulations and sensing the level of the patient's sedation and acting preemptively to either prolong or discontinue the sedation. It is an art and science that is built on years of training and experience.

The dosing of these medications is based on the patient's ideal body weight. The administration of these medications is through a continuous intravenous catheter infusion with an appropriate IV fluid. Typically, a 20- or a 22-gauge catheter is inserted into a peripheral vein in the upper extremity. Most OMSs practice the bolus technique, which introduces incremental doses of each medication, titrated to effect. Infusion pumps are often utilized in longer cases, while shorter cases of sedation seldom require infusion. 
Using a continuous infusion has the following tangible advantages:

- Minimize fluctuations in drug serum concentration

- Smoother intraoperative course

- Enhanced cardiovascular stability

- Enhanced respiratory stability

- Less patient movement

- More rapid recovery

- Minimal drug utilization

\subsection{Emergency Drugs}

The ninth edition of the AAOMS Office Anesthesia Evaluation Manual [12] provides a detailed list of medications that can be used during anesthetic emergency. OMS offices are recommended to have a refrigerator designated for medications requiring cold storage. Pharmacy boards of different states control the storage and distribution of these drugs.

Listed are suggested drugs used in the event of anesthetic emergencies:

- Intravenous fluids: normal saline, lactated Ringer, etc.

- Cardiovascular medications: Oxygen, Atropine, Nitroglycerin, Dopamine, Epinephrine, Dobutamine, Ephedrine, Phenylephrine, Lidocaine, Propranolol, Procainamide, Verapamil, Amiodarone, Adenosine

- Antihypertensive medications: Diazoxide, Hydralazine, Esmolol, Labetalol

- Diuretics: Furosemide (Lasix)

- Antiemetics: Prochlorperazine, Ondansetron

- Reversal agents: Naloxone (Narcan), Flumazenil (Romazicon)

\section{Additional Drugs}

- Dextrose 50\%, Dexamethasone (Decadron), Hydrocortisone sodium succinate or Methyl prednisolone sodium succinate (Solu-Medrol), Glycopyrolate (Robinul), Diazepam (Valium), Midazolam (Versed), Albuterol inhaler, Succinylcholine (Anectine), Morphine sulfate, Dantrolene (Dantrium), Lidocaine, Nonenteric-coated aspirin, Famotidine (Pepcid), Diphenhydramine (Benadryl)

\subsection{Activism and Leadership in Office-Based Anesthesia}

Dental anesthesia is an ADA-recognized specialty in the US as of 2019. Several dental practitioners, including OMSs, practice anxiolysis and sedation in their offices, outside a hospital operating suite and without a separate anesthesia provider. The concerted efforts by various interested parties to politically organize and better protect our intellectual and political interests in the discipline make this practice a reality in the US.

To ensure the commitment to patient safety, the AAOMS and its Board of Trustees have embraced a multipronged approach:

- undiluted enforcement of educational standards that requires resident rotation on the medical anesthesia service

- a self-imposed mandatory AAOMS Office Anesthesia Evaluation Program

- a devotion to the anesthesia team approach and development of the DAANCE program for the assistants

- the development of anesthesia and emergency management simulation programs such as Basic Emergency Airway Management [BEAM] and Office-Based Crisis Management [OBCM] are some examples of some of this commitment.

The AAOMS developed an Anesthesia Safety Conference for our own specialty that was held on April 27, 2017, at the American Society of Anesthesiologists headquarters in Schaumburg, Illinois. Representatives of the ASA as well as representatives of the appropriate disciplines of dentistry attended this meeting. In April 2019, a second patient safety conference focusing on pediatric sedation was organized at the AAOMS headquarters in Rosemont, Illinois.

By staying on the forefront of office-based anesthesia and by enforcing the highest standards of safety in patient care, OMS as a specialty has become stalwart in this arena. Continued positive momentum with nationwide simulation workshops and a mobile application supporting the office anesthesia evaluation will enhance our role as leaders in ambulatory anesthesia in our offices. The rapid response and emergency system and the healthcare infrastructure in the US allows for OMSs in this country to adapt this technique and methodology into their practices almost effortlessly. In other parts of the world, this may not be a model that is easy to replicate.

\section{Video Links}

https://myoms.org/procedures/anesthesia

\section{References}

1. American Society of Anesthesiologists (ASA). Continuum of depth of sedation: definition of general anesthesia and levels of sedation/ analgesia. (Approved by the ASA House of Delegates on October 13, 1999 and last amended on October 15, 2014). Available at: https://www.asahq.org/standards-and-guidelines/continuum-of- 
depth-of-sedation-definition-of-general-anesthesia-and-levels-ofsedationanalgesia

2. Bicentenary of nitrous oxide. Br Med J. 1972 May 13;2(5810):367368. PubMed PMID: 4554156; PubMed Central PMCID: PMC1788276.

3. West JB. Humphry Davy, nitrous oxide, the pneumatic institution, and the royal institution. Am J Physiol Lung Cell Mol Physiol 2014 Nov 1;307(9):L661-L667.

4. Desai MS, Desai SP. Discovery of modern anesthesia: a counterfactual narrative about Crawford W. long, Horace Wells, Charles T. Jackson, and William T. G. Morton. AANA J 2015 Dec;83(6):410-415. PubMed PMID: 26742335.

5. Hubbell AO, Adams CR. Intravenous anesthesia for dental surgery with sodium ethyl (1-Methylbutyl) Thiobarbituric acid. J ADA. 1940;27(8):1186-91.

6. Shane SM. Intravenous amnesia for total dentistry in one sitting. J Oral Surg 1966 Jan;24(1):27-32. PubMed PMID: 5214559.

7. Lieblich SE. Methohexital versus propofol for outpatient anesthesia. Part I: Methohexital is superior. J Oral Maxillofac Surg 1995 Jul;53(7):811-815. Review. PubMed PMID: 7595796.

8. Dembo JB. Methohexital versus propofol for outpatient anesthesia. Part II: Propofol is superior. J Oral Maxillofac Surg 1995 Jul;53(7):816-820. Review. PubMed PMID: 7595797.

9. Lieblich S. Providing anesthesia in the Oral and maxillofacial surgery office: a look Back, where we are now and a look ahead. J Oral Maxillofac Surg 2018 May;76(5):917-925. Review.

10. Drew SJ. Oral and maxillofacial surgery team anesthesia model and anesthesia assistant training. Oral Maxillofac Surg Clin North Am 2018 May;30(2):145-153. Review.

11. Chung WL. Anesthesia equipment for the oral and maxillofacial surgery practice. Oral Maxillofac Surg Clin North Am 2013 Aug;25(3):373-383.

12. American Association of Oral and Maxillofacial Surgeons (AAOMS). Office anesthesia evaluation manual. 9th edition. 2018 Available at: http://www.aaoms.org.

13. American Association of Oral and Maxillofacial Surgeons (AAOMS). Parameters of Care: AAOMS Clinical Practice
Guidelines for Oral and Maxillofacial Surgery (AAOMS ParCare) Sixth Edition 2017. Patient assessment. Available at: https://www. aaoms.org/images/uploads/pdfs/parcare_assessment.pdf

14. American Association of Oral and Maxillofacial Surgeons (AAOMS). Parameters of Care: AAOMS Clinical Practice Guidelines for Oral and Maxillofacial Surgery (AAOMS ParCare) Sixth Edition 2017. Anesthesia in outpatient facilities. Available at: https://www.joms.org/article/S0278-2391(17)30488-3/pdf

15. Todd DW, Schaefer JJ 3rd. The American Association of Oral and Maxillofacial Surgeons Simulation Program. Oral Maxillofac Surg Clin North Am 2018 May;30(2):195-206. Review.

16. ASA Physical Status Classification System. Developed By: ASA House of Delegates/Executive Committee, Last Amended: October 15, 2014 (original approval: October 15, 2014). Available at: https://www.asahq.org/standards-and-guidelines/ asa-physical-status-classification-system.

17. American Society of Anesthesiologists (ASA). Standards for basic anesthetic monitoring. Developed By: Committee on Standards and Practice Parameters (CSPP) Last Amended: October 28, 2015 (original approval: October 21, 1986). Available at: https://www.asahq.org/standards-and-guidelines/ standards-for-basic-anesthetic-monitoring.

18. Farish SE, Garcia PS. Capnography primer for Oral and maxillofacial surgery: review and technical considerations. J Anesth Clin Res 2013 Mar 18;4(3):295. PubMed PMID: 24459603; PubMed Central PMCID: PMC3897173.

\section{Additional Reading}

White Paper by American Association of Oral and Maxillofacial Surgeons (AAOMS). Office-Based Anesthesia Provided by the Oral and Maxillofacial Surgeon. 2016. Available at: https://www.aaoms. org/docs/govt_affairs/advocacy_white_papers/advocacy_office_ based_anesthesia_whitepaper.pdf

Open Access This chapter is licensed under the terms of the Creative Commons Attribution 4.0 International License (http://creativecommons. org/licenses/by/4.0/), which permits use, sharing, adaptation, distribution and reproduction in any medium or format, as long as you give appropriate credit to the original author(s) and the source, provide a link to the Creative Commons license and indicate if changes were made.

The images or other third party material in this chapter are included in the chapter's Creative Commons license, unless indicated otherwise in a credit line to the material. If material is not included in the chapter's Creative Commons license and your intended use is not permitted by statutory regulation or exceeds the permitted use, you will need to obtain permission directly from the copyright holder. 